\title{
Two Kinds of Laplacian Spectra and Degree Kirchhoff Index of the Weighted Corona Networks
}

\author{
Haiqin Liu $\mathbb{i D}^{1,2}$ and Yanling Shao $\mathbb{D}^{3}$ \\ ${ }^{1}$ Data Science and Technology, North University of China, Taiyuan, Shanxi 030051, China \\ ${ }^{2}$ Basic Department, Shanxi Agricultural University, Taigu, Shanxi 030801, China \\ ${ }^{3}$ School of Science, North University of China, Taiyuan, Shanxi 030051, China \\ Correspondence should be addressed to Yanling Shao; ylshao@nuc.edu.cn
}

Received 8 November 2021; Accepted 18 January 2022; Published 10 February 2022

Academic Editor: Azhar Hussain

Copyright (C) 2022 Haiqin Liu and Yanling Shao. This is an open access article distributed under the Creative Commons Attribution License, which permits unrestricted use, distribution, and reproduction in any medium, provided the original work is properly cited.

\begin{abstract}
Recently, the study related to network has aroused wide attention of the scientific community. Many problems can be usefully represented by corona graphs or networks. Meanwhile, the weight is a vital factor in characterizing some properties of real networks. In this paper, we give complete information about the signless Laplacian spectra of the weighted corona of a graph $G_{1}$ and a regular graph $G_{2}$ and the complete information about the normalized Laplacian spectra of the weighted corona of two regular graphs. The corresponding linearly independent eigenvectors of all these eigenvalues are also obtained. The spanning trees' total number and the degree Kirchhoff index of the weighted corona graph are computed.
\end{abstract}

\section{Introduction}

In recent years, the network research has attracted more and more attention from scientific communities [1-5], for example, chemical networks, social networks, disease transmission networks, and product networks. Especially, product networks can result some larger networks than before if through some given rules. We can synthesize new compounds through product networks and so on. However, previous researchers pay more attention to the binary networks [6-8]. However, the fact is that the weighted networks which are the extension of the binary networks are more in conformity to the networks in real world. Through the weighted networks, we can model the real world much better.

The definition of the corona and the edge corona about two graphs were given in [9] and some results about the corona have been illustrated in [10]. Azari [11-13] deduced the eccentric connectivity coindex, the Mostor index, and the edge Wiener index of the corona of two graphs. Barik et al. [14] completely represented the information about the spectrum of two graphs' corona. Hou and Shiu [15] determined the eigenvalues of the edge corona graphs of two graphs and gave the eigenvectors corresponding to them. More information about the spectra of $G_{1}{ }^{\circ} G_{2}$, such as Laplacian spectra and signless Laplacian spectra, were depicted in [16-18]. Chen and Liao [19] gave a complete description of the normalized Laplacian spectra of the corona and edge corona of two graphs.

Liu et al. [20] determined the normalized Laplacian spectra of a linear octagonal-quadrilateral network and computed the number of spanning trees of the octagonalquadrilateral networks. The Kirchhoff index, multiplicative degree Kirchhoff index, and number of spanning trees of the linear crossed hexagonal chains were obtained in [21]. Recently, Dai et al. [22] deduced the complete conclusion of generalized adjacency eigenvalues and generalized Laplacian eigenvalues of the weighted corona graph with different structures. Liu et al. [23] determined the generalized adjacency, Laplacian, and signless Laplacian spectra of the weighted edge corona networks.

Motivated by this, we study two kinds of Laplacian spectra (the signless Laplacian spectrum and the normalized Laplacian spectrum) of the weighted corona of two graphs. 
We calculate the spanning trees' number and the degree Kirchhoff index of the weighted corona graph as the application of the normalized Laplacian spectrum.

In this paper, we only pay attention to simple graphs. Let $G$ be a simple graph with vertex set $V=\left\{v_{1}, v_{2}, \ldots, v_{n}\right\}$. Let $D(G)$ denote the diagonal degree matrix of $G$. Let $A(G)$ be the adjacency matrix of $G$. It is defined as $A(G)=\left(a_{i j}\right)_{n}$, where $a_{i j}=1$ if vertices $i$ and $j$ are adjacent in $G$ and 0 otherwise.

The signless Laplacian matrix of $G$ is $Q(G)=D(G)+A(G)$. The signless Laplacian spectrum of the graph $G$ is actually the spectrum of $Q(G)$. It is denoted by

$$
S(G)=\left(\xi_{1}(G), \xi_{2}(G), \ldots, \xi_{n}(G)\right),
$$

where $\xi_{1}(G) \leq \xi_{2}(G) \leq \cdots \leq \xi_{n}(G)$ are the eigenvalues of $Q(G)$.

The normalized Laplacian matrix of $G$ is defined as

$$
\mathscr{L}(G)=D(G)^{-1 / 2}(D(G)-A(G)) D(G)^{-1 / 2},
$$

which is closely correlated with spectrum geometry and the simple random walk on $G$. Given a simple graph $G$, let $P(G)=D(G)^{-1} A(G)$ be the matrix about the simple random walk on $G$ (which is named the probability transition matrix); then,

$$
\begin{aligned}
\mathscr{L}(G) & =D(G)^{1 / 2}\left(I-D(G)^{-1} A(G)\right) D(G)^{-1 / 2} \\
& =D(G)^{1 / 2}(I-P(G)) D(G)^{-1 / 2} .
\end{aligned}
$$

So, if the spectrum of $\mathscr{L}(G)$ is denoted by

$$
N(G)=\left(\eta_{1}(G), \eta_{2}(G), \ldots, \eta_{n}(G)\right),
$$

where $\quad 0=\eta_{1}(G) \leq \eta_{2}(G) \leq \cdots \leq \eta_{n}(G) \leq 2 \quad$ and $\eta_{i}(G), i=1,2, \ldots, n$, are the eigenvalues of $\mathscr{L}(G)$. The spectrum of $P(G)$ is denoted by

$$
N p(G)=\left(\mu_{1}(G), \mu_{2}(G), \ldots, \mu_{n}(G)\right),
$$

where $1=\mu_{1}(G) \geq \mu_{2}(G) \geq \cdots \geq \mu_{n}(G) \geq-1$ and $\mu_{i}(G)$, $i=1,2, \ldots, n$, are the eigenvalues of $P(G)$. Then,

$$
\eta_{i}(G)=1-\mu_{i}(G), i=1,2, \ldots, n .
$$

More results of the normalized Laplacian spectra are depicted in $[24,25]$.

Definition 1 (see [9]). The corona of two graphs $G_{1}$ and $G_{2}$, which is denoted by $G_{1}{ }^{\circ} G_{2}$, is defined as the graph obtained by taking one copy of $G_{1}$ and $\left|V\left(G_{1}\right)\right|$ copies of $G_{2}$ and then joining the $i$ th vertex of $G_{1}$ to every vertex in the $i$ th copy of $G_{2}$.

Definition 2 (see [9]). The edge corona of two graphs $G_{1}$ and $G_{2}$, which is denoted by $G_{1} \diamond G_{2}$, is defined as the graph obtained by taking one copy of $G_{1}$ and $\left|E\left(G_{1}\right)\right|$ copies of $G_{2}$ and then joining the two vertices of each edge of graph $G_{1}$ with all vertices of corresponding copy of graph $G_{2}$ (see Figure 1).

Dai et al. have defined the weighted corona graphs of two graphs [22]. Suppose that the initial graph $G_{1}$ and copy graph $G_{2}$ are simple graphs. In the weighted corona of $G_{1}$

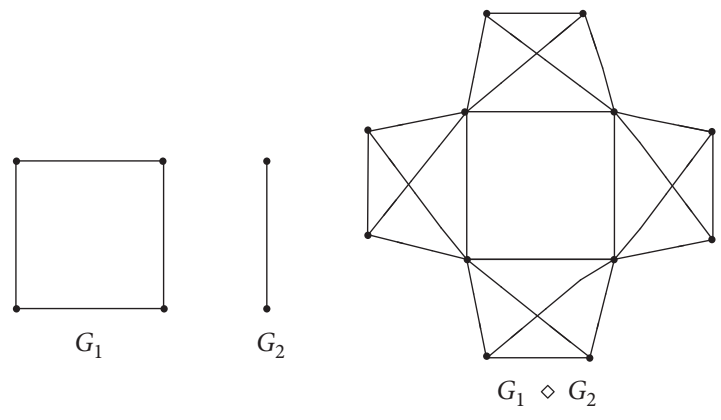

Figure 1: Edge corona $G_{1} \diamond G_{2}$ of $G_{1}$ and. $G_{2}$.

and $G_{2}$ (denoted by $\mathscr{G}=G_{1}{ }^{\circ} G_{2}$ ), each edge of $G_{1}$ has a unit weight and each edge in the copy of $G_{2}$ has a weight factor $r$, $0<r \leq 1$, and the edges which are newly generated have the weight $r$ (see Figure 2). Let $\omega_{i j}$ be the weight of edge connecting vertex $i$ and $j$ and $W(G)$ be the generalized adjacency matrix of $G$, where its element is $\omega_{i j}$ if $i$ and $j$ are adjacent, 0 otherwise. Let $A=\left(a_{i j}\right)$ and $B$ be matrices. The partition matrix $\left(a_{i j} B\right)$ is defined as the Kronecker product of $A$ and $B$, which are denoted by $A \otimes B$ [26]. If every multiplication makes sense, we can obtain

$$
\left(A_{1} \otimes A_{2}\right)\left(A_{3} \otimes A_{4}\right)=A_{1} A_{3} \otimes A_{2} A_{4} .
$$

Let $J_{n}$ denote the row vector of order $n$ with all entries equal to $1, I_{n}$ denote the identity matrix of order $n, \mathbf{0}_{n}$ denote the zero matrix of order $n$, and $e_{i}$ be the $i$ th unit column vector.

The paper is organized as below. In Section 2, the signless Laplacian spectrum of weighted corona graph is given and the corresponding linearly independent eigenvectors of all these eigenvalues are also obtained. In Section 3, the analytical formulas of the normalized Laplacian eigenvalues are determined and the corresponding linearly independent eigenvectors of all these eigenvalues are depicted. In Section 4 , we give the number of spanning trees and the degree Kirchhoff index of the weighted corona graph. Finally, we draw the conclusion.

\section{The Signless Spectrum of Weighted Corona Graph $G_{1}{ }^{\circ} G_{2}$}

The strength of vertex $i$ is $\tilde{d}_{i}=\sum_{j \in G} \omega_{i j}(i=1,2, \ldots, n)$. Let $\widetilde{D}(G)=\operatorname{diag}\left(\widetilde{d}_{1}, \widetilde{d}_{2}, \ldots, \widetilde{d}_{n}\right)$ denote the diagonal intensity matrix of $G$. Then, the signless Laplacian matrix of the weighted graph $\mathscr{G}$ is defined by $Q(\mathscr{G})=\widetilde{D}(\mathscr{G})+W(\mathscr{G})$. The spectrum of $Q(\mathscr{G})$ is said to be the signless Laplacian spectrum of $\mathscr{G}$. In this section, we will give the signless Laplacian eigenvalues of the weighted corona graph $\mathscr{G}=G_{1}{ }^{\circ} G_{2}$.

Let $G_{1}$ be a graph with $n_{1}$ vertices and $G_{2}$ be a $k$-regular graph with $n_{2}$ vertices. Then, the signless Laplacian matrix of the weighted corona graph $G_{1}{ }^{\circ} G_{2}$ is as follows:

$$
Q\left(G_{1}{ }^{\circ} G_{2}\right)=\left(\begin{array}{cc}
Q\left(G_{1}\right)+r n_{2} I_{n_{1}} & r J_{n_{2}} \otimes I_{n_{1}} \\
r J_{n_{2}}^{T} \otimes I_{n_{1}} & r\left(Q\left(G_{2}\right)+I_{n_{2}}\right) \otimes I_{n_{1}}
\end{array}\right),
$$




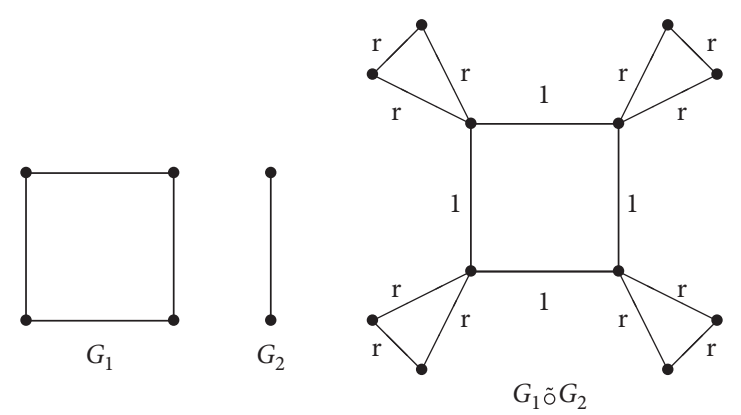

FIgURE 2: The weighted corona graph, $G_{1}{ }^{\circ} G_{2}$.

where $Q\left(G_{1}\right)$ and $Q\left(G_{2}\right)$ are the signless Laplacian matrices of the graphs $G_{1}$ and $G_{2}$, respectively.

Theorem 1. Let $G_{1}$ be a graph with $n_{1}$ vertices and $G_{2}$ be a $k$-regulargraph with $n_{2}$ vertices, and $S\left(G_{1}\right)=\left(\mu_{1}, \mu_{2}, \ldots, \mu_{n_{1}}\right)$ and $S\left(G_{2}\right)=\left(\eta_{1}, \eta_{2}, \ldots, \eta_{n_{2}}=2 k\right)$. Then, the signless Laplacian spectra of the weighted corona $G_{1}{ }^{\circ} G_{2}$ are as follows.

(i) $\mu_{i}+r n_{2}+(2 k+1) r \pm$

$$
\begin{array}{ll} 
& \sqrt{\left[\mu_{i}+r n_{2}+(2 k+1) r\right]^{2}-4(2 k+1) r} \\
& \mu_{i}-8 r^{2} n_{2} k / 2 \in S\left(G_{1}{ }^{\circ} G_{2}\right) \quad \text { with multiplicity } 1 \text { for } \\
& i=1,2, \ldots, n_{1} \\
\text { (ii) } & r\left(\eta_{j}+1\right) \in S\left(G_{1}{ }^{\circ} G_{2}\right) \quad \text { with multiplicity } n_{1} \text { for } \\
& j=1,2, \ldots, n_{2}-1
\end{array}
$$

Proof. Let $\lambda$ be the eigenvalue of $Q\left(G_{1}{ }^{\circ} G_{2}\right)$, $X=\left(X_{1} X_{2} \cdots X_{n_{2}+1}\right)^{T}$, and $X_{i} \in R^{n_{1}}\left(i=1,2, \ldots, n_{2}+1\right)$ be the eigenvector which corresponds to $\lambda$. Then,

$$
Q\left(G_{1}{ }^{\circ} G_{2}\right) X=\lambda X \text {. }
$$

From equations (8) and (9), we can conclude that

$$
\left(Q\left(G_{1}\right)+r n_{2} I_{n_{1}}\right) X_{1}^{T}+r\left(X_{2}^{T}+X_{3}^{T}+\cdots+X_{n_{2}+1}^{T}\right)=\lambda X_{1}^{T},
$$

and

$$
\left\{\begin{array}{l}
\operatorname{lr} X_{1}^{T}+r E_{1}\left(\left(Q\left(G_{2}\right)+I_{n_{2}}\right) \otimes I_{n_{1}}\right)\left(X_{2} X_{3} \ldots X_{n_{2}+1}\right)^{T}=\lambda X_{2}^{T}, \\
r X_{1}^{T}+r E_{2}\left(\left(Q\left(G_{2}\right)+I_{n_{2}}\right) \otimes I_{n_{1}}\right)\left(X_{2} X_{3} \ldots X_{n_{2}+1}\right)^{T}=\lambda X_{3}^{T}, \\
\vdots \\
r X_{1}^{T}+r E_{n_{2}}\left(\left(Q\left(G_{2}\right)+I_{n_{2}}\right) \otimes I_{n_{1}}\right)\left(X_{2} X_{3} \ldots X_{n_{2}+1}\right)^{T}=\lambda X_{n_{2}+1}^{T},
\end{array}\right.
$$

where $E_{i}=\left(\sim \mathbf{0}_{n_{1}}{ }^{i-1}, \ldots, \mathbf{0}_{n_{1}}, I_{n_{1}}, \sim \mathbf{0}_{n_{1}}, \ldots, \mathbf{0}_{n_{1}}\right)$.

By adding the above equations in equation (11), we have

$$
\begin{aligned}
& r n_{2} X_{1}^{T}+(2 k+1) r\left(X_{2}^{T}+X_{3}^{T}+\cdots+X_{n_{2}+1}^{T}\right) \\
& =\lambda\left(X_{2}^{T}+X_{3}^{T}+\cdots+X_{n_{2}+1}^{T}\right),
\end{aligned}
$$

which is

$$
r n_{2} X_{1}^{T}=[\lambda-(2 k+1) r]\left(X_{2}^{T}+X_{3}^{T}+\cdots+X_{n_{2}+1}^{T}\right) .
$$

Case 1: $X_{1}$ is the nonzero vector.

In this case, we immediately deduce that $\lambda-(2 k+1) r \neq 0$. From equations (10) and (13), it gives

$$
\left(Q\left(G_{1}\right)+r n_{2} I_{n_{1}}\right) X_{1}^{T}+\frac{r^{2} n_{2}}{\lambda-(2 k+1) r} X_{1}^{T}=\lambda X_{1}^{T},
$$

so

$$
Q\left(G_{1}\right) X_{1}^{T}=\left[\lambda-r n_{2}-\frac{r^{2} n_{2}}{\lambda-(2 k+1) r}\right] X_{1}^{T} .
$$

Noticing that $S\left(G_{1}\right)=\left(\mu_{1}, \mu_{2}, \ldots, \mu_{n_{1}}\right)$ and according to equation (15), one obtains

$$
\lambda-r n_{2}-\frac{r^{2} n_{2}}{\lambda-(2 k+1) r}=\mu_{i}, \quad i=1,2, \ldots, n_{1},
$$

which is

$$
\begin{aligned}
& \lambda^{2}-\left[(2 k+1) r+r n_{2}+\mu_{i}\right] \lambda+2 r^{2} n_{2} k \\
& +(2 k+1) r \mu_{i}=0, \quad i=1,2, \ldots, n_{1} .
\end{aligned}
$$

By solving this equation about $\lambda$, we obtain

$$
\begin{aligned}
& \lambda_{i}, \bar{\lambda}_{i}=\frac{\mu_{i}+r n_{2}+(2 k+1) r \pm \sqrt{\left[\mu_{i}+r n_{2}+(2 k+1) r\right]^{2}-4(2 k+1) r \mu_{i}-8 r^{2} n_{2} k}}{2} . \\
& \text { zero vector. } \\
& \qquad X_{1}^{T}+X_{2}^{T}+\cdots+X_{n_{2}+1}^{T}=\mathbf{0},
\end{aligned}
$$

Case 2: $X_{1}$ is the zero vector.

and 


$$
\begin{aligned}
& r\left(Q\left(G_{2}\right)+I_{n_{2}}\right) \otimes I_{n_{1}}\left(X_{2} X_{3} \ldots X_{n_{2}+1}\right)^{T} \\
& =\lambda\left(X_{2} X_{3} \ldots X_{n_{2}+1}\right)^{T} .
\end{aligned}
$$

Note that $(2 k+1) r$ is not the eigenvalue of $Q\left(G_{1}{ }^{\circ} G_{2}\right)$. Otherwise, we can deduce that the eigenvector $X=\left[X_{1} X_{2} \ldots X_{k+1}\right]^{T}$ of the eigenvalue $(2 k+1) r$ fits $X^{T}=J_{n_{1}} \otimes J_{n_{2}+1}$. Thus,

$$
X_{1}+X_{2}+\cdots+X_{n_{2}+1}=\left(n_{2}+1\right) J_{n_{1}},
$$

which contradicts with equation (19).

Note that the spectrum of $Q\left(G_{2}\right)$ is $S\left(G_{2}\right)=\left(\eta_{1}, \eta_{2}, \ldots, \eta_{n_{2}}=2 k\right)$, so according to the equation (20), we can directly obtain

$$
\lambda=r\left(\eta_{j}+1\right), \quad j=1,2, \ldots, n_{2}-1,
$$

and the multiplicity of $\lambda=r\left(\eta_{j}+1\right)$ is $n_{1}$.

According to equations (18) and (22), we have $n_{1}\left(1+n_{2}\right)$ eigenvalues of $Q\left(G_{1}{ }^{\circ} G_{2}\right)$. The eigenvectors of $Q\left(G_{1}{ }^{\circ} G_{2}\right)$ corresponding to these eigenvalues are presented as follows.

From equation (15), we obtain that $X_{1}^{T}$ is the eigenvector set corresponding to the eigenvalues of $Q\left(G_{1}\right)$. Let $X_{1}=Y_{i}, X_{2}=X_{3}=\cdots=X_{n_{2}+1}=r Y_{i} / \lambda_{i}-(2 k+1) r$, and $X_{2}^{\prime}=X_{3}^{\prime}=\cdots=X_{n_{2}+1}{ }^{\prime}=r Y_{i} / \lambda_{i}-(2 k+1) r \quad$ and $\quad Y_{i}(i=$ $\left.1,2, \ldots, n_{1}\right)$ be the nonzero eigenvectors corresponding to the eigenvalues $\mu_{1}, \mu_{2}, \ldots, \mu_{n_{1}}$. Let $F_{i}=\left(X_{1} X_{2} \ldots X_{n_{2}+1}\right)^{T}$ and $F_{i}^{\prime}=\left(X_{1} X_{2}^{\prime} \ldots X_{n_{2}+1}\right)^{T}$, for $i=1,2, \ldots, n_{1}$. We need to prove $Q\left(G_{1}{ }^{\circ} G_{2}\right) F_{i}=\lambda_{i} F_{i}$ and $Q\left(G_{1}{ }^{\circ} G_{2}\right) F_{i}^{\prime}=\bar{\lambda}_{i} F_{i}^{\prime}$ in order to confirm that $F_{i}$ and $F_{i}^{\prime}$ are $2 n_{1}$ eigenvectors corresponding to $\lambda_{i}$ and $\bar{\lambda}_{i}$, respectively.

Given a vertex $u$ in $G_{1}{ }^{\circ} G_{2}$. If $u \in V\left(G_{1}\right)$, one has $F_{i}(u)=Y_{i}(u)$, and it obtains

$$
\begin{aligned}
& \left(Q\left(G_{1}\right)+r n_{2} I_{n_{1}} r J n_{2} \otimes I_{n_{1}}\right) F_{i}(u) \\
& =Q\left(G_{1}\right) Y_{i}(u)+r n_{2} Y_{i}(u)+\frac{r^{2} n_{2} Y_{i}(u)}{\lambda_{i}-(2 k+1) r} \\
& =\mu_{i} Y_{i}(u)+r n_{2} Y_{i}(u)+\frac{r^{2} n_{2} Y_{i}(u)}{\lambda_{i}-(2 k+1) r} \\
& =\lambda_{i} Y_{i}(u) .
\end{aligned}
$$

If $u$ is a vertex in the $t$ th copy of $G_{2}$, one has $F_{i}(u)=r Y_{i}(t) / \lambda_{i}-(2 k+1) r$, and it obtains

$$
\begin{aligned}
& \left(r I_{n_{1}} r E_{t}\left(\left(Q\left(G_{2}\right)+I_{n_{2}}\right) \otimes I_{n_{1}}\right)\right) F_{i}(u) \\
& =r Y_{i}(t)+\frac{(2 k+1) r^{2}}{\lambda_{i}-(2 k+1) r} Y_{i}(t) \\
& =\lambda_{i} F_{i}(u) .
\end{aligned}
$$

For each vertex in $G_{1}{ }^{\circ} G_{2}$, it follows that $Q\left(G_{1}{ }^{\circ} G_{2}\right) F_{i}=\lambda_{i} F_{i}$. Similarly, for each vertex in $G_{1}{ }^{\circ} G_{2}$, we have $Q\left(G_{1}{ }^{\circ} G_{2}\right) F_{i}^{\prime}=\bar{\lambda}_{i} F_{i}^{\prime}$.

Let $Z_{j}\left(j=1,2, \ldots, n_{2}\right)$ be the orthogonal eigenvectors of $Q\left(G_{2}\right)$ corresponding to $\eta_{1}, \eta_{2}, \ldots, \eta_{n_{2}}=2 k$. Noticing that $Z_{n_{2}}=a J_{n_{2}}^{T}$ and $Z_{j} \perp J_{n_{2}}^{T}$, for $j=1,2, \ldots, n_{2}-1$. So, for $i=1,2, \ldots, n_{1}$ and $j=1,2, \ldots, n_{2}-1$, one knows

$$
\begin{aligned}
Q\left(\tilde{G_{1}} G_{2}\right)\left(\begin{array}{c}
\mathbf{0}_{n_{1}} \\
Z_{j} \otimes e_{i}
\end{array}\right) & =\left(\begin{array}{c}
\mathbf{0}_{n_{1}} \\
\left(r\left(Q\left(G_{2}\right)+I_{n_{2}}\right) \otimes I_{n_{1}}\right)\left(Z_{j} \otimes e_{i}\right)
\end{array}\right) \\
& =\left(\begin{array}{c}
\mathbf{0}_{n_{1}} \\
\left(r\left(Q\left(G_{2}\right)+I_{n_{2}}\right) Z_{j} \otimes I_{n_{1}} e_{i}\right.
\end{array}\right) \\
& =r\left(\eta_{j}+1\right)\left(\begin{array}{c}
\mathbf{0}_{n_{1}} \\
Z_{j} \otimes e_{i}
\end{array}\right) .
\end{aligned}
$$

Now, we have obtained $n_{1}\left(1+n_{2}\right)$ eigenvectors of $Q\left(G_{1}{ }^{\circ} G_{2}\right)$, and they are linearly independent obviously.

\section{The Normalized Laplacian Spectrum of Weighted Corona Graph $G_{1}{ }^{\circ} G_{2}$}

Let $G_{1}$ be an $r_{1}$-regular graph with $n_{1}$ vertices and $G_{2}$ an $r_{2}$-regular graph with $n_{2}$ vertices. Let $\mathscr{G}=G_{1}{ }^{\circ} G_{2}$. Then,

$$
\begin{aligned}
W(\mathscr{G}) & =\left(\begin{array}{cc}
W\left(G_{1}\right) & r J_{n_{2}} \otimes I_{n_{1}} \\
r J_{n_{2}}^{T} \otimes I_{n_{1}} & r W\left(G_{2}\right) \otimes I_{n_{1}}
\end{array}\right), \\
\widetilde{D}(\mathscr{G}) & =\left(\begin{array}{cc}
\left(r_{1}+r n_{2}\right) I_{n_{1}} & 0 \\
0 & r\left(r_{2}+1\right) I_{n_{1} n_{2}}
\end{array}\right), \\
P(\mathscr{G}) & =\widetilde{D}(\mathscr{G})^{-1} W(\mathscr{G}) \\
& =\left(\begin{array}{cc}
\frac{1}{r_{1}+r n_{2}} W\left(G_{1}\right) & \frac{r}{r_{1}+r n_{2}} J_{n_{2}} \otimes I_{n_{1}} \\
\frac{1}{r_{2}+1} J_{n_{2}}^{T} \otimes I_{n_{1}} & \frac{1}{r_{2}+1} W\left(G_{2}\right) \otimes I_{n_{1}}
\end{array}\right) \\
& =\left(\begin{array}{cc}
\frac{r}{r_{1}+r n_{2}} P\left(G_{1}\right) & \frac{r}{r_{1}+r n_{2}} J_{n_{2}} \otimes I_{n_{1}} \\
\frac{1}{r_{2}+1} J_{n_{2}}^{T} \otimes I_{n_{1}} & \frac{r_{2}}{r_{2}+1} P\left(G_{2}\right) \otimes I_{n_{1}}
\end{array}\right) .
\end{aligned}
$$

Theorem 2. Let $G_{1}$ be an $r_{1}$-regular graph with $n_{1}$ vertices and $G_{2}$ an $r_{2}$-regular graph with $n_{2}$ vertices. The spectra of $P\left(G_{1}\right)$ and $P\left(G_{2}\right)$ are

$N_{p}\left(G_{1}\right)=\left(\beta_{1}, \beta_{2}, \ldots, \beta_{n_{1}}\right)$ and $N_{p}\left(G_{2}\right)=\left(\rho_{1}, \rho_{2}, \ldots, \rho_{n_{2}}\right)$,

respectively, where $-1=\beta_{1} \leq \beta_{2} \leq \cdots \leq \beta_{n_{1}}=1$ and $-1=\rho_{1} \leq \rho_{2} \leq \cdots \leq \rho_{n_{2}}=1$. Then, the spectra of $P\left(G_{1}{ }^{\circ} G_{2}\right)$ are as follows:

(i) $r_{2}\left(r_{1}+r n_{2}\right)+r_{1} \beta_{i}\left(r_{2}+1\right) \pm \sigma_{i} / 2\left(r_{1}+r n_{2}\right)\left(r_{2}+\right.$ 1) $\in N_{p}\left(G_{1}{ }^{\circ} G_{2}\right)$ with multiplicity 1 , for $i=1,2, \ldots, n_{1}$, where

$\sqrt{\sigma_{i}=}=$ 
(ii) $r_{2} \rho_{j} / r_{2}+1 \in N_{p}\left(G_{1}{ }^{\circ} G_{2}\right)$ with multiplicity $n_{1}$, for $j=1,2, \ldots, n_{2}-1$

Proof. Let $\lambda$ be the eigenvalue of $P(\mathscr{G})$, $X=\left(X_{1} X_{2} \ldots X_{n_{2}+1}\right)^{T}$, and $X_{i} \in R^{n_{1}}\left(i=1,2, \ldots, n_{2}+1\right)$ be the eigenvector which corresponds to $\lambda$. Then,

$$
P(\mathscr{G}) X=\lambda X \text {. }
$$

From equations (28) and (30), it follows that

$$
\begin{aligned}
& r_{1} / r_{1}+r n_{2} P\left(G_{1}\right) X_{1}^{T}+r / r_{1} \\
& +r n_{2}\left(X_{2}^{T}+X_{3}^{T}+\cdots+X_{n_{2}+1}^{T}\right)=\lambda X_{1}^{T}
\end{aligned}
$$

and

$$
\left\{\begin{array}{l}
l \frac{1}{r_{2}+1} X_{1}^{T}+\frac{r_{2}}{r_{2}+1} E_{1}\left(P\left(G_{2}\right) \otimes I_{n_{1}}\right)\left(X_{2} X_{3} \ldots X_{n_{2}+1}\right)^{T}=\lambda X_{2}^{T}, \\
\frac{1}{r_{2}+1} X_{1}^{T}+\frac{r_{2}}{r_{2}+1} E_{2}\left(P\left(G_{2}\right) \otimes I_{n_{1}}\right)\left(X_{2} X_{3} \ldots X_{n_{2}+1}\right)^{T}=\lambda X_{3}^{T}, \\
\vdots \\
\frac{1}{r_{2}+1} X_{1}^{T}+\frac{r_{2}}{r_{2}+1} E_{n_{2}}\left(P\left(G_{2}\right) \otimes I_{n_{1}}\right)\left(X_{2} X_{3} \ldots X_{n_{2}+1}\right)^{T}=\lambda X_{n_{2}+1}^{T},
\end{array}\right.
$$

where $E_{i}=\left(-\mathbf{0}_{n_{1}}{ }^{i-1}, \ldots, \mathbf{0}_{n_{1}}, I_{n_{1}}, \cdots \mathbf{0}_{n_{1}}, \ldots, \mathbf{0}_{n_{1}}\right)$.

By equation (32), we obtain

$$
\begin{aligned}
& \frac{n_{2}}{r_{2}+1} X_{1}^{T}+\frac{r_{2}}{r_{2}+1}\left(X_{2}^{T}+X_{3}^{T}+\cdots+X_{n_{2}+1}^{T}\right) \\
& =\lambda\left(X_{2}^{T}+X_{3}^{T}+\cdots+X_{n_{2}+1}^{T}\right),
\end{aligned}
$$

that is,

$\frac{n_{2}}{r_{2}+1} X_{1}^{T}+\left(\frac{r_{2}}{r_{2}+1}-\lambda\right)\left(X_{2}^{T}+X_{3}^{T}+\cdots+X_{n_{2}+1}^{T}\right)=0$.

Case 1: $X_{1}$ is the nonzero vector.

$\frac{r_{1}}{r_{1}+r n_{2}} P\left(G_{1}\right) X_{1}^{T}+\frac{r}{r_{1}+r n_{2}} \frac{n_{2}}{\lambda r_{2}+\lambda-r_{2}} X_{1}^{T}=\lambda X_{1}^{T}$,

In this case, we immediately deduce that $r_{2} / r_{2}+1-\lambda \neq 0$. From equations (31) and (34), we have so

$$
P\left(G_{1}\right) X_{1}^{T}=\frac{1}{r_{1}}\left[\lambda\left(r_{1}+r n_{2}\right)-\frac{r n_{2}}{\lambda r_{2}+\lambda-r_{2}}\right] X_{1}^{T} .
$$

Noting that $N_{p}\left(G_{1}\right)=\left(\beta_{1}, \beta_{2}, \ldots, \beta_{n_{1}}\right)$ and according to equation (36), we can obtain

$\lambda\left(\left(r_{1}+r n_{2}\right)-\frac{r n_{2}}{\lambda r_{2}+\lambda-r_{2}}=r_{1} \beta_{i}, \quad i=1,2, \ldots, n_{1}\right.$.

By solving this equation about $\lambda$, we obtain

$$
\begin{aligned}
\lambda_{i}, \bar{\lambda}_{i} & =\frac{r_{2}\left(r_{1}+r n_{2}\right)+r_{1} \beta_{i}\left(r_{2}+1\right) \pm \sigma_{i}}{2\left(r_{1}+r n_{2}\right)\left(r_{2}+1\right)}, \\
\sigma_{i} & =\sqrt{\left[r_{2}\left(r_{1}+r n_{2}\right)-r_{1} \beta_{i}\left(r_{2}+1\right)\right]^{2}+4 r n_{2}\left(r_{1}+r n_{2}\right)\left(r_{2}+1\right)} .
\end{aligned}
$$

Case 2: $X_{1}$ is the zero vector.

From equations (31) and (32), we obtain

$$
X_{2}^{T}+\cdots+X_{n_{2}+1}^{T}=\mathbf{0}
$$

and

$\frac{r_{2}}{r_{2}+1} P\left(G_{2}\right) \otimes I_{n}\left(X_{2} X_{3} \ldots X_{n_{2}+1}\right)^{T}=\lambda\left(X_{2} X_{3} \ldots X_{n_{2}+1}\right)^{T}$.
Notice that $r_{2} / r_{2}+1$ is not the eigenvalue of $P\left(G_{1}{ }^{\circ} G_{2}\right)$. Otherwise, we can deduce that the eigenvector $X=\left(X_{1} X_{2} \ldots X_{n_{2}+1}\right)^{T}$ of the eigenvalue $r_{2} / r_{2}+1$ fits $X^{T}=J_{n_{1}} \otimes J_{n_{2}+1}$. Thus,

$$
X_{1}+X_{2}+\cdots+X_{n_{2}+1}=\left(n_{2}+1\right) J_{n},
$$

which contradicts with equation (39). Note that the spectrum of $P\left(G_{2}\right)$ is $N_{p}\left(G_{2}\right)=\left(-1=\rho_{1} \leq \rho_{2} \leq \cdots \leq \rho_{n_{2}}=1\right)$, so according to equation (40), we can directly obtain 


$$
\lambda=\frac{r_{2} \rho_{j}}{r_{2}+1}, \quad j=1,2, \ldots, n_{2}-1,
$$

and the multiplicity of $\lambda=r_{2} \rho_{j} / r_{2}+1$ is $n_{1}$.

According to equations (38) and (42), we have $n_{1}\left(1+n_{2}\right)$ eigenvalues of $P\left(G_{1}{ }^{\circ} G_{2}\right)$. The eigenvectors of $P\left(G_{1}{ }^{\circ} G_{2}\right)$ which corresponding to these eigenvalues are presented as follows.

From equation (36), we obtain that $X_{1}^{T}$ is the eigenvector set corresponding to the eigenvalues of $P\left(G_{1}\right)$. Let $X_{1}=Y_{i}, X_{2}=X_{3}=\cdots=X_{n_{2}+1}=r Y_{i} / \lambda_{i}\left(r_{2}+1\right)-r_{2}$, and $X_{2}^{\prime}=X_{3}^{\prime}=\cdots=X_{n_{2}+1}{ }^{\prime}=r Y_{i} / \lambda_{i}\left(r_{2}+1\right)-r_{2}$, and $Y_{i}(i=$ $\left.1,2, \ldots, n_{1}\right)$ be the nonzero eigenvectors which correspond to the eigenvalues $\mu_{1}, \mu_{2}, \ldots, \mu_{n_{1}}$. Let $F_{i}=\left(X_{1} X_{2} \ldots X_{n_{2}+1}\right)^{T}$ and $F_{i}^{\prime}=\left(X_{1} X_{2}^{\prime} \ldots X_{n_{2}+1}\right)^{T}$, for $i=1,2, \ldots, n_{1}$. We need to prove $P\left(G_{1}{ }^{\circ} G_{2}\right) F_{i}=\lambda_{i} F_{i}$ and $P\left(G_{1}{ }^{\circ} G_{2}\right) F_{i}^{\prime}=\bar{\lambda}_{i} F_{i}^{\prime}$ in order to confirm that $F_{i}$ and $F_{i}^{\prime}$ are $2 n_{1}$ eigenvectors corresponding to $\lambda_{i}$ and $\bar{\lambda}_{i}$, respectively.

Given a vertex $u$ in $G_{1}{ }^{\circ} G_{2}$. If $u \in V\left(G_{1}\right)$, it obtain

$$
\begin{aligned}
( & \left.\frac{r_{1}}{r_{1}+r n_{2}} P\left(G_{1}\right) \frac{r}{r_{1}+r n_{2}} J_{n_{2}} \otimes I_{n_{1}}\right) F_{i}(u) \\
= & \frac{r_{1}}{r_{1}+r n_{2}} P\left(G_{1}\right) Y_{i}(u) \\
& +\frac{r n_{2}}{\left(r_{1}+r n_{2}\right)\left(\lambda_{i}\left(r_{2}+1\right)-r_{2}\right)} Y_{i}(u) \\
= & \frac{r_{1}}{r_{1}+r n_{2}} \beta_{i} Y_{i}(u)+\frac{r n_{2}}{\left(r_{1}+r n_{2}\right)\left(\lambda_{i}\left(r_{2}+1\right)-r_{2}\right)} Y_{i}(u) \\
= & \lambda_{i} F_{i}(u) .
\end{aligned}
$$

If $u$ is a vertex in one copy of $G_{2}$, one has $F_{i}(u)=Y_{i}(t) / \lambda_{i}\left(r_{2}+1\right)-r_{2}$, and it obtains

$$
\begin{aligned}
& \left(\frac{1}{r_{2}+1} I_{n_{1}} \frac{r_{2}}{r_{2}+1} E_{t}\left(P\left(G_{2}\right) \otimes I_{n_{1}}\right)\right) F_{i}(u) \\
& =\frac{1}{r_{2}+1} Y_{i}(t)+\frac{r_{2}}{\left(r_{2}+1\right)\left(\lambda_{i}\left(r_{2}+1\right)-r_{2}\right)} Y_{i}(t) \\
& =\lambda_{i} F_{i}(u) .
\end{aligned}
$$

For each vertex in $G_{1}{ }^{\circ} G_{2}$, it follows that $P\left(G_{1}{ }^{\circ} G_{2}\right) F_{i}(u)=\lambda_{i} F_{i}(u)$. Similarly, for each vertex in $G_{1}{ }^{\circ} G_{2}$, we have $P\left(G_{1}{ }^{\circ} G_{2}\right) F_{i}(u)=\bar{\lambda}_{i} F_{i}(u)$.

Let $Z_{j}\left(j=1,2, \ldots, n_{2}\right)$ be the orthogonal eigenvectors of $P\left(G_{2}\right)$ corresponding to $\rho_{1}, \rho_{2}, \ldots, \rho_{n_{2}}=2 k$. Noticing that $Z_{n_{2}}=a J_{n_{2}}^{T}$ and $Z_{j} \perp J_{n_{2}}^{T}$, for $j=1,2, \ldots, n_{2}-1$. So, for $i=1,2, \ldots, n_{1}$ and $j=1,2, \ldots, n_{2}-1$, one knows

$$
\begin{aligned}
P\left(\tilde{G_{1}} G_{2}\right)\left(\begin{array}{c}
\mathbf{0}_{n_{1}} \\
Z_{j} \otimes e_{i}
\end{array}\right) & =\left(\begin{array}{c}
\mathbf{0}_{n_{1}} \\
\left.r_{2} / r_{2}+1 P\left(G_{2}\right) \otimes I_{n_{1}}\right)\left(Z_{j} \otimes e_{i}\right)
\end{array}\right) \\
& =\left(\begin{array}{c}
\mathbf{0}_{n_{1}} \\
r_{2} / r_{2}+1 P\left(G_{2}\right) Z_{j} \otimes I_{n_{1}} e_{i}
\end{array}\right) \\
& =\frac{r_{2} \rho_{j}}{r_{2}+1}\left(\begin{array}{c}
\mathbf{0}_{n_{1}} \\
Z_{j} \otimes e_{i}
\end{array}\right) .
\end{aligned}
$$

Now, we have obtained $n_{1}\left(1+n_{2}\right)$ eigenvectors of $P\left(G_{1}{ }^{\circ} G_{2}\right)$, and they are linearly independent obviously. We easily deduce the following result according to relation (6).

Theorem 3. Let $G_{1}$ be an $r_{1}$-regular graph with $n_{1}$ vertices and $G_{2}$ an $r_{2}$-regular graph with $n_{2}$ vertices. The spectra of $\mathscr{L}\left(G_{1}\right)$ and $\mathscr{L}\left(G_{2}\right)$ are

$$
\begin{aligned}
N\left(G_{1}\right) & =\left(0=\gamma_{1}, \gamma_{2}, \ldots, \gamma_{n_{1}}\right) \text { and } N\left(G_{2}\right) \\
& =\left(0=\delta_{1}, \delta_{2}, \ldots, \delta_{n_{2}}\right),
\end{aligned}
$$

respectively. Then, the normalized Laplacian spectra of $G_{1}{ }^{\circ} G_{2}$ are as follows:

(i) $r n_{2}\left(r_{2}+2\right)+r_{1}+\gamma_{i} r_{1}\left(r_{2}+1\right) \pm \tau_{i} / 2\left(r_{1}+r n_{2}\right)\left(r_{2}+\right.$ 1) $\in N\left(G_{1}{ }^{\circ} G_{2}\right)$ with multiplicity 1 for $i=1,2, \ldots, n_{1}$, where

$$
\begin{aligned}
& \tau_{i}= \\
& \sqrt{\left[r r_{2} n_{2}-r_{1}+r_{1} \gamma_{i}\left(r_{2}+1\right)\right]^{2}+4 r n_{2}\left(r_{1}+r n_{2}\right)\left(r_{2}+1\right)} \\
& \text { (ii) } 1+r_{2} \delta_{j} / r_{2}+1 \in N\left(G_{1}{ }^{\circ} G_{2}\right) \text { with multiplicity } n_{1} \text { for } \\
& \quad j=1,2, \ldots, n_{2}-1
\end{aligned}
$$

\section{Application}

In this section, the spanning trees' number and the degree Kirchhoff index of the weighted corona graph are obtained as applications of Theorem 2.

Given a graph $G$, the spanning tree is the tree which contains all vertices of $G$. Therefore, there are many spanning trees of graph $G$ due to the complex structure of the tree. Let the graph $G$ be a connected graph, the number of vertices be $n$, and the number of edges be $m$. The normalized Laplacian eigenvalues of $G$ is $0=\lambda_{1} \leq \lambda_{2} \leq \lambda_{3} \leq \ldots \leq \lambda_{n}$. Then, the number of spanning tree of $G$ [21] can be expressed by 


$$
t(G)=\prod_{i \in V(G)} d_{i} \frac{\prod_{j=2}^{n} \lambda_{j}}{2 m,}
$$

where $d_{i}$ is the degree of vertex $i$.

In [25], Chen and Zhang proposed a new graph invariant (called the degree Kirchhoff index) which is defined as $K f(G)=\sum_{i<j} d_{i} d_{j} r_{i j}$. They have proved that this graph invariant is associated with the normalized Laplacian eigenvalues of $G$, that is,

$$
K f(G)=2 m \sum_{j=2}^{n} \frac{1}{\lambda_{j}} .
$$

In the following theorem, we will give the number of spanning trees of the weighted corona graph $G_{1}{ }^{\circ} G_{2}$ and its degree Kirchhoff index, by using the normalized Laplacian spectrum of graph $G_{1}{ }^{\circ} G_{2}$.

Theorem 4. Let $G_{1}$ be an $r_{1}$-regular graph with $n_{1}$ vertices and $G_{2}$ an $r_{2}$-regular graph with $n_{2}$ vertices. Then, for the weighted corona $\mathscr{G}=G_{1}{ }^{\circ} G_{2}$, we have

(i) $t(\mathscr{G})=r^{n_{1} n_{2}}\left(r r_{2} n_{2}+2 r n_{2}+r_{1}\right) / r_{1}+r_{2} n_{2}+$

$2 n_{2} t\left(G_{1}\right) \prod_{j=1}^{n_{2}-1}\left(r_{2} \delta_{j}+1\right)^{n_{1}}$

(ii) $K f(\mathscr{G})=\left(r r_{2} n_{2}+2 r n_{2}+r_{1}\right)\left(r_{2} n_{2}+2 n_{2}+r_{1}\right)$

$/ r_{1}^{2} K f \quad\left(G_{1}\right)+2|E(\mathscr{G})|\left(r_{2}+1\right)\left(r_{1}+r n_{2} / r r_{2} n_{2}+\right.$ $\left.2 r n_{2}+r_{1}+n_{1}-1+\sum_{j=1}^{n_{2}-1} n_{1} / r_{2} \delta_{j}+1\right)$, where $\delta_{j}$ is the normalized Laplacian eigenvalue of $G_{2}$

Proof. Let the normalized Laplacian spectra of $G_{1}$ and $G_{2}$ be as depicted in Theorem 2. Then, by Theorem 2, $\gamma_{1}=0$ corresponds two eigenvalues 0 and $r r_{2} n_{2}+2 r n_{2}+r_{1} /\left(r_{1}+\right.$ $\left.r n_{2}\right)\left(r_{2}+1\right)$ of $\mathscr{L}(\mathscr{G})$; for each $\gamma_{i} \neq 0$, the product of the two corresponding eigenvalues is

$$
\begin{aligned}
& \frac{\left[r n_{2}\left(r_{2}+2\right)+r_{1}+\gamma_{i} r_{1}\left(r_{2}+1\right)\right]^{2}-\tau_{i}^{2}}{4\left(r_{1}+r n_{2}\right)^{2}\left(r_{2}+1\right)^{2}} \\
& =\frac{r_{1} \gamma_{i}}{\left(r_{1}+r n_{2}\right)\left(r_{2}+1\right)} .
\end{aligned}
$$

So,

$$
\begin{aligned}
\prod_{\lambda \in L(G), \lambda \neq 0} \lambda= & \frac{r r_{2} n_{2}+2 r n_{2}+r_{1}}{\left(r_{1}+r n_{2}\right)\left(r_{2}+1\right)} \prod_{i=2}^{n_{1}} \\
& \frac{r_{1} \gamma_{i}}{\left(r_{1}+r n_{2}\right)\left(r_{2}+1\right)} \prod_{j=1}^{n_{2}-1}\left(\frac{r_{2} \delta_{j}+1}{r_{2}+1}\right)^{n_{1}} \\
= & \frac{\left(r r_{2} n_{2}+2 r n_{2}+r_{1}\right) r_{1}^{n_{1}-1} \prod_{i=2}^{n_{1}} \gamma_{i} \prod_{j=1}^{n_{2}-1}\left(r_{2} \delta_{j}+1\right)^{n_{1}}}{\left(r_{1}+r n_{2}\right)^{n_{1}}\left(r_{2}+1\right)^{n_{1} n_{2}}} .
\end{aligned}
$$

Hence,

$$
\begin{aligned}
t(\mathscr{G}) & =\frac{\left(r_{1}+r n_{2}\right)^{n_{1}}\left(r\left(r_{2}+1\right)\right)^{n_{1} n_{2}} \prod_{\lambda \in L(G), \lambda \neq 0} \lambda}{n_{1}\left(r_{1}+r_{2} n_{2}+2 n_{2}\right)} \\
& =\frac{r^{n_{1} n_{2}}\left(r r_{2} n_{2}+2 r n_{2}+r_{1}\right)}{r_{1}+r_{2} n_{2}+2 n_{2}} \frac{r_{1}^{n_{1}} \prod_{i=2}^{n_{1}} \gamma_{i}}{r_{1} n_{1}} \prod_{j=1}^{n_{2}-1}\left(r_{2} \delta_{j}+1\right)^{n_{1}} \\
& =\frac{r^{n_{1} n_{2}}\left(r r_{2} n_{2}+2 r n_{2}+r_{1}\right)}{r_{1}+r_{2} n_{2}+2 n_{2}} t\left(G_{1}\right) \prod_{j=1}^{n_{2}-1}\left(r_{2} \delta_{j}+1\right)^{n_{1}} .
\end{aligned}
$$

For the Kirchhoff index, we have

$$
\begin{aligned}
K f(\mathscr{G}) & =2|E(\mathscr{G})| \sum_{\lambda \in L(\mathscr{G}), \lambda \neq 0} \frac{1}{\lambda} \\
& =2|E(\mathscr{G})|\left(\frac{\left(r_{1}+r n_{2}\right)\left(r_{2}+1\right)}{r r_{2} n_{2}+2 r n_{2}+r_{1}}+\sum_{i=2}^{n_{1}} \frac{r n_{2}\left(r_{2}+2\right)+r_{1}+\gamma_{i} r_{1}\left(r_{2}+1\right)}{\gamma_{i} r_{1}}+\sum_{j=1}^{n_{2}-1} \frac{n_{1}\left(r_{2}+1\right)}{r_{2} \delta_{j}+1}\right) \\
& =\frac{\left(r r_{2} n_{2}+2 r n_{2}+r_{1}\right)\left(r_{2} n_{2}+2 n_{2}+r_{1}\right)}{r_{1}^{2}} K f\left(G_{1}\right)+2|E(\mathscr{G})|\left(r_{2}+1\right)\left(\frac{r_{1}+r n_{2}}{r r_{2} n_{2}+2 r n_{2}+r_{1}}+n_{1}-1+\sum_{j=1}^{n_{2}-1} \frac{n_{1}}{r_{2} \delta_{j}+1}\right) .
\end{aligned}
$$

\section{Conclusion}

In recent decades, the study of the normalized Laplacian spectrum has more practical significance, for some dynamical processes and structural aspects of various networks are related to it. This study explores the signless Laplacian spectrum and the normalized Laplacian spectrum of the weighted corona graphs.

\section{Data Availability}

Data sharing is not applicable to this article as no new data were created or analyzed in this study.

\section{Conflicts of Interest}

The authors declare that they have no conflicts of interest. 


\section{Acknowledgments}

This work was supported by the Shanxi Province Science Foundation for Youths (no. 201901D211227).

\section{References}

[1] M. F. Dai, X. Wang, J. Zou, Y. Chen, and W. Su, "First-order network coherence and eigentime identity on the weighted Cayley networks," Fractals, vol. 25, no. 5, Article ID 1750049, 2017.

[2] F. Ma and B. Yao, "The relations between network-operation and topological-property in a scale-free and small-world network with community structure," Physica A: Statistical Mechanics and Its Applications, vol. 484, pp. 182-193, 2017.

[3] M. F. Dai, J. Zou, and X. Wang, "Eigentime identity of the weighted Koch networks," Fractals, vol. 26, no. 3, Article ID 185004, 2018.

[4] J. Cao, J. B. Liu, and S. Wang, "Resistance distances in corona and neighborhood corona networks based on Laplacian generalized inverse spproach," Journal of Algebra and Its Applications, vol. 18, no. 03, Article ID 195005, 2019.

[5] J. Yao, B. Sun, and 1. Xi, "Fractality of evolving self-similar networks," Physica A: Statistical Mechanics and Its Applications, vol. 515, pp. 211-216, 2019.

[6] K. Avrachenkov and I. Bogdanov, "Analysis of relaxation time in random walk with jumps," in International Workshop on Algorithms and Models for the Web-GraphSpringer, Berlin, Germany, 2018.

[7] C. Cooper, A. Frieze, and T. Johansson, The Cover Time Os a Biased Random Walk on a Random Cubic Graph, 2018.

[8] Q. Ding, W. Sun, and F. Chen, "Network coherence in the web graphs, Commun," Communications in Nonlinear Science and Numerical Simulation, vol. 27, no. 1-3, pp. 228-236, 2015.

[9] F. Harary, Graph Theory, Addition-Wesley Publishing Co., Reading, MA/Menlo Park, CA/London, 1969.

[10] R. Frucht and F. Harary, "On the corona two graphs," Aequationes Mathematicae, vol. 4, no. 3, pp. 322-325, 1970.

[11] M. Azari, "Eccentric connectivity coindex under graph operations," Journal of Applied Mathematics and Computing, vol. 62, no. 1-2, pp. 23-35, 2020.

[12] Y. Alizadeh, A. Iranmanesh, T. Došlić, and M. Azari, “The edge Wiener index of suspensions, bottlenecks, and thorny graphs," Glasnik Matematicki Series III, vol. 49, no. 69, pp. 1-12, 2014.

[13] M. Azari and N. Dehgardi, "Measuring peripherality extent in chemical graphs via graph op- erations," International Journal of Quantum Chemistry, vol. 122, no. 3, Article ID e26835, 2021.

[14] S. Barik, S. Pati, and B. K. Sarma, "The spectrum of the corona of two graphs," SIAM Journal on Discrete Mathematics, vol. 21, no. 1, pp. 47-56, 2007.

[15] Y. Hou and W. Shiu, "The spectrum of the edge corona of two graphs," Electronic Journal of Linear Algebra, vol. 20, pp. 586-594, 2010.

[16] S.-Y. Cui and G.-X. Tian, "The spectrum and the signless Laplacian spectrum of coronae," Linear Algebra and Its Applications, vol. 437, no. 7, pp. 1692-1703, 2012.

[17] S. Y. Cui and G. X. Tian, "The signless Laplacian spectrum of the(edge) corona of two graphs," Utilitas Mathematica, vol. 88, pp. 287-297, 2012.

[18] S. Wang and B. Zhou, "The signless Laplacian spectra of the corona and edge corona of two graphs," Linear and Multilinear Algebra, vol. 61, no. 2, pp. 197-204, 2013.
[19] H. Chen and L. Liao, "The normalized Laplacian spectra of the corona and edge corona of two graphs," Linear and Multilinear Algebra, vol. 65, no. 3, pp. 582-592, 2017.

[20] J. B. Liu, J. Zhao, and Z. X. Zhu, "On the number of spanning trees and normalized Laplacian of linear octagonal-quadrilateral networks," International Journal of Quantum Chemistry, vol. 119, no. 17, Article ID e25971, 2019.

[21] Y. Pan and J. Li, "Kirchhoff index, multiplicative degreeKirchhoff index and spanning trees of the linear crossed hexagonal chains," International Journal of Quantum Chemistry, vol. 118, no. 24, Article ID e25787, 2018.

[22] M. Dai, J. Shen, L. Dai, T. Ju, Y. Hou, and W. Su, "Generalized adiacency and Laplician spectra of the weighted corona graphs," Physica A: Statistical Mechanics and Its Applications, vol. 528, Article ID 121285, 2019.

[23] J.-B. Liu, J. Zhao, and Z.-Q. Cai, "On the generalized adjacency, Laplacian and signless Laplacian spectra of the weighted edge corona networks," Physica A: Statistical Mechanics and Its Applications, vol. 540, Article ID 123073, 2020.

[24] F. R. K. Chung, "Spectral graph theory. CBMS. Regional conference series in mathematics," Providence: AMS, vol. 92, 1997.

[25] H. Chen and F. Zhang, "Resistance distance and the normalized Laplacian spectrum," Discrete Applied Mathematics, vol. 155, no. 5, pp. 654-661, 2007.

[26] R. A. Horn and C. R. Johnson, Topics in Matrix Analysis, Cambridge University Press, Cambridge, UK, 1991. 